NORDITA 95/47-N, nucl-th/9507023

\title{
Kaon Energies in Dense Matter
}

\author{
V. R. Pandharipande ${ }^{1}$, C. J. Pethick ${ }^{1,2,3}$ and Vesteinn Thorsson ${ }^{2}$, \\ ${ }^{1}$ Department of Physics, University of Illinois at Urbana-Champaign, \\ 1110 West Green St., Urbana, Illinois 61801-3080, USA \\ ${ }^{2}$ NORDITA, Blegdamsvej 17, DK-2100 Copenhagen Ø, Denmark \\ ${ }^{3}$ Institute for Nuclear Theory, University of Washington, Box 351550, Seattle, Washington \\ 98195-1550, USA
}

(September 7, 2018)

\begin{abstract}
We discuss the role of kaon-nucleon and nucleon-nucleon correlations in kaon condensation in dense matter. Correlations raise the threshold density for kaon condensation, possibly to densities higher than those encountered in stable neutron stars.
\end{abstract}

PACS numbers: 13.75.Jz, 21.65.+f, 97.60.Jd

Typeset using REVTEX 
The possibility of kaon condensation in dense matter was suggested by Kaplan and Nelson [1], and it has been discussed in many recent publications. [2 77]. The basic idea is that the energy of a $K^{-}, \epsilon_{K}$, is lowered by interaction with nucleons, and in neutron star matter in beta equilibrium one expects negative kaons to be present if the energy to create one in the medium is less than the electron chemical potential, $\mu_{e}$, the energy required to add an extra electron.

One complication in determining the low energy interactions of kaons with nucleons is that the interactions of kaons with protons in vacuo is dominated by the $\Lambda(1405)$ resonance. Fortunately this does not affect the $K^{-}$interaction with neutrons, which is expected to dominate in neutron stars. In dense matter the effects of the $\Lambda(1405)$ are likely unimportant, because the kinematic region of interest is far from the resonance. To describe the $K N$ interactions Brown, Lee, Rho, and Thorsson [5], herafter denoted by BLRT, employed an effective Lagrangian based on chiral perturbation theory. To circumvent the difficulties with the resonance, parameters of the Lagrangian were fitted to $K^{+} N,(N=n$ or $p)$, data, which are unaffected by the resonance. The Lagrangian was treated in mean field theory, and the density for condensation was found to be $\sim 3-4 \rho_{0}$, where $\rho_{0}=0.16 \mathrm{fm}^{-3}$ is nuclear matter density. This is to be compared with the central density of about $4 \rho_{0}$ for a neutron star of mass $1.4 M_{\odot}$ according to the estimates of Wiringa, Fiks and Fabrocini [8] using realistic models of nuclear forces. The conclusion was that kaon condensation could affect the structure, maximum mass and cooling rates of neutron stars significantly.

In this Letter, we first show that an important contribution to the kaon energy in the BLRT treatment is the first term in an expansion in powers of the particle density, with a strength proportional to kaon-nucleon scattering lengths. We then argue that kaon-nucleon correlations in the medium will be reduced compared with those for a kaon and nucleon in vacuo, and that consequently the attraction experienced by a kaon in matter will be smaller that earlier estimates indicated.

BLRT use the Lagrangian of Jenkins and Manohar [9] containing terms of the two lowest orders in the chiral expansion, and the nucleons are treated in mean field theory, that is 
their densities are assumed to be uniform, and equal to $\rho_{n}$ and $\rho_{p}$. The spatially uniform state, which is the one of lowest energy in this model, is given by

$$
K^{ \pm}=v_{K} e^{ \pm i \epsilon_{K} t}
$$

and

$$
\begin{array}{r}
\mathcal{L}=(1-\alpha) \dot{K}^{+} \dot{K}^{-}-\left(m_{K}^{2}-\delta^{2}\right) K^{+} K^{-} \\
-\frac{i}{2} V\left(K^{-} \dot{K}^{+}-\dot{K}^{-} K^{+}\right),
\end{array}
$$

with $V=\left(\rho_{n}+2 \rho_{p}\right) /\left(2 f^{2}\right), \delta^{2}=\left[\Sigma_{K N}\left(\rho_{n}+\rho_{p}\right)-C\left(\rho_{n}-\rho_{p}\right)\right] / f^{2}$, and $\alpha=-\left[\tilde{D}\left(\rho_{n}+\rho_{p}\right)-\right.$ $\left.\tilde{D}^{\prime}\left(\rho_{n}-\rho_{p}\right)\right] / f^{2}$. The $V$ and $\delta^{2}$ terms describe interactions of kaons with effective vector and scalar fields, while $\alpha$ takes into account energy dependence of the interaction. The pion decay constant, $f(\approx 93 \mathrm{MeV})$, and the constant $C(\approx 33.5 \mathrm{MeV})$ are well determined, but the values of $\Sigma_{K N}, \tilde{D}$, and $\tilde{D}^{\prime}$ are not well known. The experimental $K^{+} N$ scattering lengths 10 are used to determine $\tilde{D}$ for chosen values of $\Sigma_{K N}$ from Eq.(27) of BLRT. Eq.(28) of BLRT gives $\tilde{D}^{\prime}=0.092 / m_{K}$. A large $\Sigma_{K N}$ implies a large energy dependence for the $K^{-} N$ interaction, and the value $\Sigma_{K N}=278 \mathrm{MeV}$ was considered to be realistic by BLRT. It is presently considered likely that $\Sigma_{K N}$ lies around $400 \mathrm{MeV}$ [6]. The corresponding values of $\tilde{D}$ are $-0.23 / m_{K}$ for $\Sigma_{K N}=278 \mathrm{MeV}$, and $-0.48 / m_{K}$ for $\Sigma_{K N}=400 \mathrm{MeV}$.

The $K^{-}$energy may easily be calculated from Eq. (2), and is

$$
\epsilon_{K}=\frac{1}{2(1-\alpha)}\left(\left(4(1-\alpha)\left(m_{K}^{2}-\delta^{2}\right)+V^{2}\right)^{1 / 2}-V\right),
$$

as used by BLRT. Observing that $\alpha, \delta^{2}$ and $V$ are linear in the nucleon densities, $\rho_{N}$, we expand $\epsilon_{K}$ in powers of $\rho_{N}$. To second order in the densities one finds

$$
\begin{array}{r}
\epsilon_{K}=m_{K}-\left(\frac{V}{2}+\frac{\delta^{2}}{2 m_{K}}-\frac{\alpha m_{K}}{2}\right) \\
+\frac{V^{2}}{8 m_{K}}-\frac{\delta^{4}}{8 m_{K}^{3}}+\frac{3}{8} \alpha^{2} m_{K}-\frac{V \alpha}{2}-\frac{\alpha \delta^{2}}{4 m_{K}} .
\end{array}
$$

The first term is just the kaon rest mass. The second term may be expressed simply by making use of the fact that the $K^{-} N$ scattering lengths [11] are given by 


$$
\begin{aligned}
& a_{K^{-} n}=\frac{-m_{R}}{4 \pi f^{2} m_{K}}\left(\frac{m_{K}}{2}+\Sigma_{K N}-C+\left(\tilde{D}-\tilde{D}^{\prime}\right) m_{K}^{2}\right), \\
& a_{K^{-} p}=\frac{-m_{R}}{4 \pi f^{2} m_{K}}\left(m_{K}+\Sigma_{K N}+C+\left(\tilde{D}+\tilde{D}^{\prime}\right) m_{K}^{2}\right),
\end{aligned}
$$

where $m_{R}=m_{N} m_{K} /\left(m_{N}+m_{K}\right)$ is the reduced mass. This term is thus the Lenz potential,

$$
\Delta \epsilon_{K, \text { Lenz }}=\frac{2 \pi}{m_{R}}\left(\rho_{n} a_{K^{-} n}+\rho_{p} a_{K^{-} p}\right)
$$

the well-known result for the energy shift to first order in the density ( see also Ref. [7]). Thus for given values of the scattering lengths, the Lenz potential is independent of the choice of $\Sigma_{K N}$. However, terms of order $\rho_{N}^{2}$ and higher depend on $\Sigma_{K N}$. When $\Sigma_{K N}$ is small, $\delta^{2}$ and $\alpha$ are small (see Table 1) and the higher order terms dominated by $V^{2} /\left(8 m_{K}\right)$ are repulsive. In contrast, when $\Sigma_{K N}$ is large, $\delta^{2}$ is large, $1-\alpha$ can become zero at relatively small densities, $\sim 10 \rho_{0}$, and the higher order terms are attractive.

The dependence of the kaon energy on nucleon density is illustrated in Fig.1 for a proton fraction of 0.1. The curves labelled by $\Sigma_{K N}$ values are obtained from Eq.(可). For comparison, the Lenz approximation, which contains only the first two terms in Eq.(4), is also shown. The Lenz term gives a major contribution, while other terms representing the energy dependence of the interaction and relativistic effects may be significant.

The Lenz term is exact only at low density, and there are corrections to it when it is no longer a good approximation to assume that the $K^{-} N$ relative wave function, $\psi_{K^{-} N}$, is the same as for a pair of particles in vacuo. For s-wave scattering, $\psi$ at low energy is given by

$$
\psi_{K^{-} N}(r)=1-a_{K^{-} N} / r \text { for } r>R
$$

where $R$ is the range of the $K^{-} N$ interaction. The corresponding unperturbed wave function is simply unity.

To illustrate the effects, we represent the $K^{-} N$ interaction by potentials, corresponding to the two possible isospin values, $I=0,1$. The scattering lengths are given by

$$
a_{K^{-N}}=\frac{m_{R}}{2 \pi} \int d^{3} r \mathcal{V}_{K^{-N}}(r) \psi_{K^{-} N}(r)
$$


where $\mathcal{V}_{K^{-} N}(r)$ represents the $K^{-} N$ potential. Since $\psi_{K^{-} N}(r<R)>1-a_{K^{-} N} / R$ when $\mathcal{V}_{K^{-} N}(r)$ does not have a repulsive core, $2 \pi a_{K^{-N}} / m_{R}$ is larger than the volume integral of $\mathcal{V}_{K^{-} N}(r)$ by a factor larger than $1-a_{K^{-} N} / R$. The potential $\mathcal{V}_{K^{-} N}(r)$ is believed to be short ranged, because the vector and scalar mesons that contribute to it have masses larger than the inverse of hadron radii. Hence $\mathcal{V}_{K^{-} N}$ has a range $R<1 \mathrm{fm}$, and the factor $1-a_{K^{-} N} / R$ is larger than 1.41 and 1.85 for $N=n, p$ with the scattering lengths $a_{K^{-} n}=-0.41 \mathrm{fm}$ and $a_{K^{-} p}=-0.85 \mathrm{fm}$ given by Eqs.(5) and (6), and the parameters taken from BLRT. These factors represent the correlation between low-energy $K^{-}$and nucleons in vacuo, and when it is significantly different from unity, the Lenz term is valid only at densities low enough that the radius, $r_{0}$, of a sphere with a volume equal to the average volume per particle, $1 / \rho=4 \pi r_{0}^{3} / 3$ is larger than both $R$ and $|a|$.

In order to make quantitative estimates, we fitted square well and Yukawa potential to the scattering lengths. For a square well of radius $0.7 \mathrm{fm}$, the depths are $126.2 \mathrm{MeV}$ for neutrons and $181 \mathrm{MeV}$ for protons, while for $\mathrm{R}=1.0 \mathrm{fm}$, the corresponding values are 49.5 and 75.6 MeV. For a Yukawa potential $V_{0} \exp \left(-m_{x} r\right) /\left(m_{x} r\right)$, one finds $V_{0}=-795 \mathrm{MeV}$ for neutrons and $-1075 \mathrm{MeV}$ for protons if the mass, $m_{x}$, of the exchanged particle is taken to be that of the $\rho$ meson, $770 \mathrm{MeV}$. In neutron stars, it is the $K^{-} n$ interaction that is the more important, since the proton fraction of matter is small, and in Fig. 2 we show $\psi_{K^{-} n}$ for the three potentials. These exhibit the enhancement of the wave function for small separations.

At higher densities, the kaon energy in matter should be calculated either by summation of diagrams, as in approaches that evaluate the $K^{-} N$ interaction $G$ matrix and higher-order correlations, or from variational wave functions of the type

$$
\Psi=\left[\Pi_{i=1, A} f_{K^{-N}}\left(\vec{r}_{K^{-}}-\vec{r}_{i}\right)\right] \Psi_{N}\left(\vec{r}_{1}, \ldots \vec{r}_{A}\right),
$$

where $f_{K^{-} N}$ represents the $K^{-}-$nucleon correlation and $\Psi_{N}$ is the ground state of a system with $A$ nucleons. At the large densities of interest in the cores of neutron stars, $r_{0}<1 \mathrm{fm}$. When $r_{0}<R$, the kaon is weakly correlated with nucleons, and $f_{K^{-} N}$ is unity to a good approximation. In this limit the Lenz potential is replaced by the Hartree potential, and 
the $K^{-}$energy is given by

$$
\epsilon_{\text {Hartree }}=m_{K}+\int\left[\rho_{n} \mathcal{V}_{K^{-} n}(r)+\rho_{p} \mathcal{V}_{K^{-} p}(r)\right] d^{3} r
$$

The Hartree potential is less attractive than the Lenz one by a factor in excess of 1 $a_{K^{-} N} / R$. In Fig. 1 we show estimates of the Hartree contribution to the kaon energy for the potentials we fitted to the scattering lengths, as described above. The Hartree calculation does not include effects of energy dependence of the interaction or of relativity, but since the Hartree energy is closer to $m_{K}$ than is the Lenz term, we expect these effects to be smaller than estimated from Eq. (4).

To investigate the validity of the Hartree approximation we now estimate second order terms in the $K^{-} N$ interaction. For simplicity, we neglect the effects of the small number of protons. In this case, the main coupling of the kaon is to neutron density fluctuations, since the kaon is spinless, and consequently does not couple to spin fluctuations. The contribution to the energy of a kaon at rest is then given by

$$
\Delta \epsilon_{K}^{(2)}=-\sum_{\vec{q}, l}|\mathcal{V}(q)|^{2} \frac{|<l| \rho_{\vec{q}}|0>|^{2}}{\epsilon_{K}(q)-\epsilon_{K}(0)+E_{l}-E_{0}} .
$$

Here $<l\left|\rho_{\vec{q}}\right| 0>$ is the matrix element of the operator for the Fourier transform of the neutron density between the ground state, 0 , and an excited state, $l$, and $E_{0}$ and $E_{l}$ are the energies of the states. The energy of a kaon with momentum $\vec{p}$ is denoted by $\epsilon_{K}(p)$. If one neglects the recoil energy of the kaon, one finds an upper bound for the energy shift in second order:

$$
\begin{gathered}
-\Delta \epsilon_{K}^{(2)}<\frac{1}{2} \sum_{\vec{q}}|\mathcal{V}(q)|^{2} \chi(q, \omega=0) \\
=\frac{1}{2} \int d^{3} r d^{3} r^{\prime} \mathcal{V}(r) \chi\left(\vec{r}-\vec{r}^{\prime}\right) \mathcal{V}\left(r^{\prime}\right)
\end{gathered}
$$

where

$$
\chi(q, 0)=\sum_{l} 2 \frac{|<l| \rho_{\vec{q}}|0>|^{2}}{E_{l}-E_{0}} .
$$


is the static density-density response function for neutrons, and $\chi(r)$ is its Fourier transform. The recoil energy of the kaon is comparable in magnitude to the excitation energy of the neutron liquid for the wavevectors of importance, so the actual second order contribution to the energy is expected to be considerably smaller than the bound. The long-wavelength static density-density response function for neutron matter may be estimated by using the thermodynamic identity $\chi(q \rightarrow 0, \omega=0)=d \rho_{n} / d \mu_{n}$, together with many-body calculations of the neutron chemical potential as a function of density. The dependence of $\chi$ on wavenumber is not well known, and to obtain an estimate of the second-order term we shall replace $\chi(q)$ by its long-wavelength value. At a density of $4 \rho_{0}$, the estimates of the second order term obtained by using values of $d \rho_{n} / d \mu_{n}$ from Ref. [12 are $7 \mathrm{MeV}$ (square well, $R=1$ fm), $16 \mathrm{MeV}$ (square well, $R=0.7 \mathrm{fm}$ ), and $46 \mathrm{MeV}$ (Yukawa), and for higher densities the estimates are smaller. In all cases, these energies are considerably less than the Hartree potential.

The second order contribution to the energy is reduced greatly due to the strong repulsive interactions between nucleons. Had we neglected neutron-neutron interactions, the estimates of the second order term, which would then reflect Pauli blocking of intermediate states in the repeated scattering of a kaon by a neutron [13], would have been much greater. For a free Fermi gas, $\rho_{n} d \mu_{n} / d \rho_{n}=2 E_{F} / 3$, where $E_{F} \approx 58\left(\rho_{n} / \rho_{0}\right)^{2 / 3} \mathrm{MeV}$ is the Fermi energy. At a density of $4 \rho_{0}, \rho_{n} d \mu_{n} / d \rho_{n}$ estimated from the calculations of Friedman and Pandharipande [12 is about 5 times larger than the free gas value. The fact that stable neutron stars with a mass of $1.4 M_{\odot}$ exist is a clear indication that the equation of state of neutron star matter is considerably stiffer than that for a free Fermi gas. While there are still uncertainties about the properties of matter at high densities, this increased stiffness is a rather general property shared by many models, as one can see from, e.g., Ref. [8]. As a first step towards a more complete many-body treatment, we are calculating the kaon energy using the lowest order constrained variational (LOCV) method [14], and the results will be reported elsewhere.

To explore consequences of our calculations for kaon condensation, we also show in Fig.1 
the electron chemical potential, $\mu_{e}$, calculated by Wiringa et al. [8]. Our Hartree estimates of kaon energies always lie above $\mu_{e}$ for essentially all stable neutron star models. However, for a number of reasons we are prevented from definitively ruling out kaon condensation in neutron stars. First, we have used simple approximate forms for the kaon-nucleon interactions, and estimates should be made with better forms of the interaction, which should also take into account the variation of the coupling constant, $f$, with nuclear density [5]. Second, corrections to the Hartree result need to be investigated in detail. Third, as one can see from the figure, estimates of $\mu_{e}$ at high densities are subject to considerable uncertainty: models of nucleon interactions that fit laboratory data equally well lead to very different values of $\mu_{e}$ in neutron star matter. All these points need to be looked into.

The main conclusion of our calculations is that, in a dense medium such as that in the interiors of neutron stars, correlations are very different from those for a dilute gas. This has the effect of reducing the attraction experienced by a kaon in dense matter. While a low-order chiral expansion may be useful for describing low-energy scattering of kaons by nucleons, this information alone can predict the kaon energy only at low densities. When the $K^{-} N$ scattering lengths are as large as indicated by BLRT, the Lenz approximation overestimates the attraction felt by a $K^{-}$in neutron star matter at the density of neutron star cores by roughly a factor of 2 .

This work was supported in part by NSF grants NSF PHY94-21309 and NSF AST9315133, and NASA grant NAGW-1583. We are grateful to D. G. Ravenhall, W. Weise, and R. B. Wiringa for helpful discussions. One of us (CJP) is grateful to the Institute for Nuclear Theory at the University of Seattle for hospitality during the workshop there on chiral symmetry, when he received partial support from the Department of Energy grant to the Institute. 


\section{REFERENCES}

* Address after Sept. 15, 1995: Department of Physics, University of Washington, Box 351560, Seattle, Washington 98195-1560, USA.

[1] D. B. Kaplan and A. E. Nelson, Phys. Lett. B175, 57 (1986).

[2] G. E. Brown, K. Kubodera, M. Rho and V. Thorsson, Phys. Lett. B291, 355 (1992)

[3] T. Muto and T. Tatsumi, Phys. Lett. B283, 165 (1992). W. Weise, Nucl. Phys. A553, 59c (1993). M. Lütz, A. Steiner and W. Weise A574, 755 (1994). J. Schaffner, A. Gal, I. N. Mishustin, H. Stöcker, and W. Greiner, Phys. Lett. B334, 268 (1994). V. Koch, Phys. Lett. B377, 7 (1994).

[4] V. Thorsson, M. Prakash and J. M. Lattimer, Nucl. Phys. A572, 693 (1994).

[5] G. E. Brown, C.-H. Lee, M. Rho and V. Thorsson, Nucl. Phys. A567, 937 (1994).

[6] C.-H. Lee, G. E. Brown, D.-P. Min and M. Rho, Nucl. Phys. A585, 401 (1995).

[7] V. Thorsson and A. Wirzba, Preprint Nordita-95/7 N, nucl-th/9502003, Nucl. Phys. A, in print.

[8] R. B. Wiringa, V. Fiks and A. Fabrocini, Phys. Rev. C38, 1010 (1988).

[9] E. Jenkins and A. Manohar, Phys. Lett. B255, 558 (1991). E. Jenkins, Nucl. Phys. B368, 190 (1991).

[10] T. Barnes and E. S. Swanson, Phys. Rev. C49, 1166 (1994).

[11] We caution the reader that, in order to make the paper accessible to most physicists, we use the usual sign convention for scattering lengths, with positive scattering lengths corresponding to repulsive potentials. This is the opposite of the convention commonly employed in the literature on kaon-nucleon and pion-nucleon interactions.

[12] B. Friedman and V. R. Pandharipande, Nucl. Phys. A361, 502 (1981). 
[13] Estimates of the effects of Pauli blocking at low densities may be found in C. B. Dover and P. J. Moffa, Phys. Rev. C16, 1087 (1977) and in Ref.[6] .

[14] V. R. Pandharipande and H. A. Bethe, Phys. Rev. C7, 1312 (1973); V. R. Pandharipande and K. E. Schmidt, Phys. Rev. A15, 2486 (1977). 


\section{FIGURES}

FIG. 1. Energy of a single $K^{-}$in matter as a function of density, and electron chemical potentials taken from the calculations of Ref.[6]. The calculations of kaon energies are for a proton fraction of 0.1. Curves labelled by values of $\Sigma_{K N}$, in $\mathrm{MeV}$, are evaluated from Eq.(3). Hartree potentials, evaluated from Eq.(11), are shown for square wells of radii $R=1 \mathrm{fm}$ and $R=0.7$ fm, and for a Yukawa potential. A and U refer to the AV14 and UV14 two-body potentials, and VII and TNI to the UVII and TNI many-body potentials. The open circles indicate the central densities of neutron stars with a mass of $1.4 M_{\odot}$, and filled circles indicate the central density for the most massive stable neutron star for the given equation of state.

FIG. 2. Wave function for the relative motion of a $\mathrm{K}^{-}$and a neutron at zero energy for square well potentials (solid lines) and a Yukawa potential ( long-dashed line). The short-dashed line shows the asymptotic limit of the wave functions. 


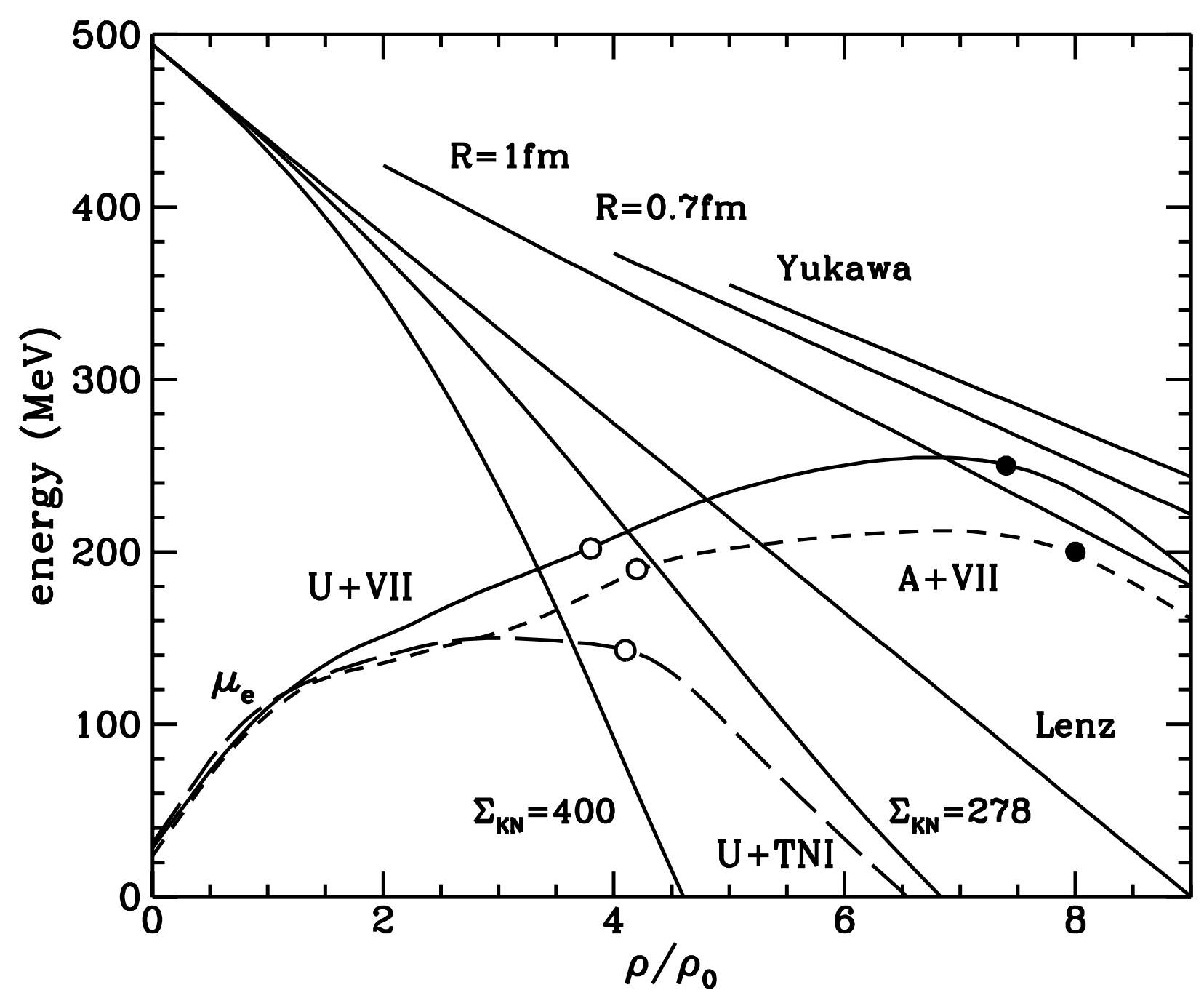

Fig.1, Pandharipande et al., Kaon Energies in Dense Matter 


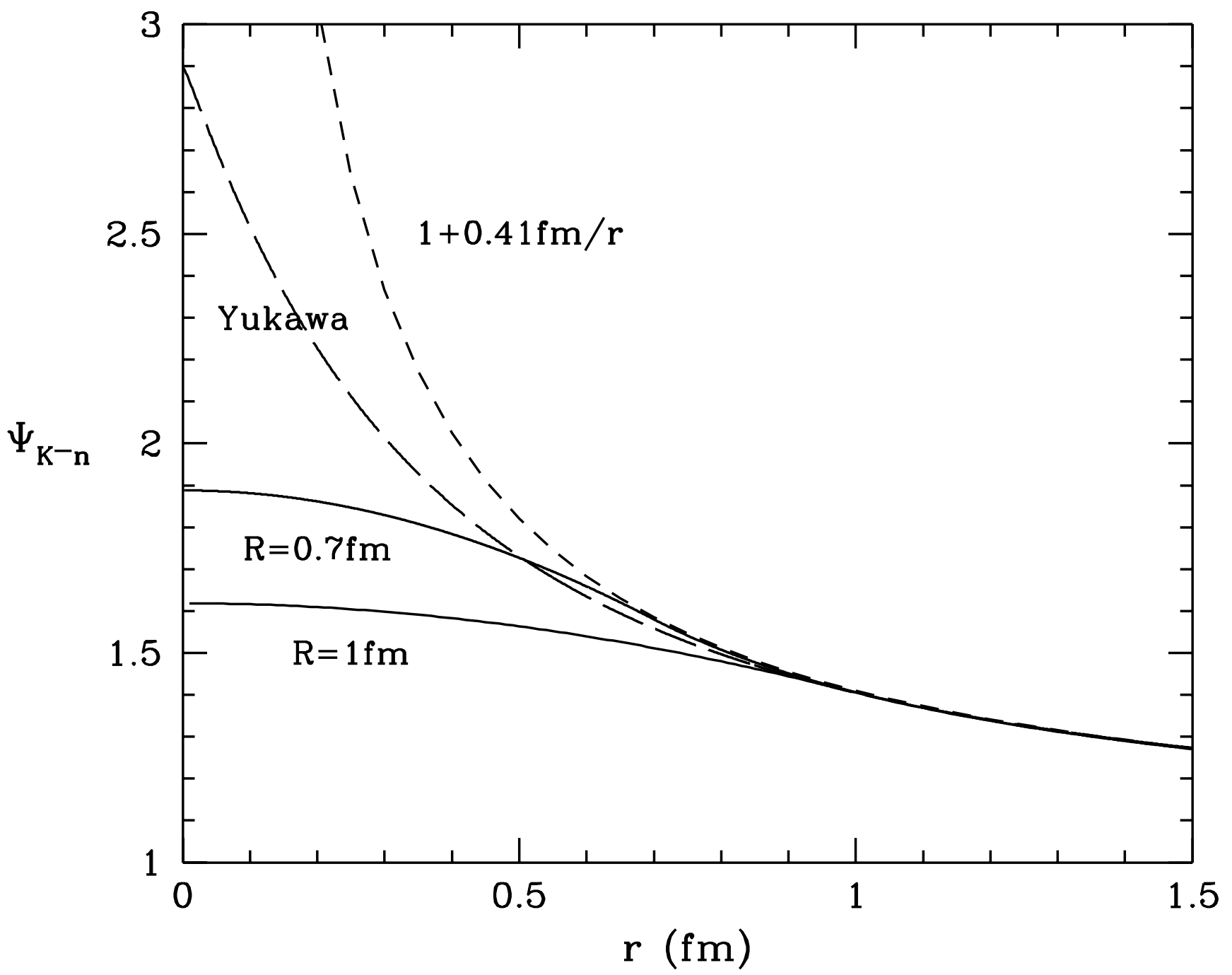

Fig.2, Pandharipande et al., Kaon Energies in Dense Matter 